\title{
On the Semantic Attrition of High Degree Adverb in Mandarin Chinese
}

\author{
Yong Yang ${ }^{1,2}$ \\ ${ }^{1}$ Research Center for Language and Language Education, Central China Normal University, Wuhan, \\ China, 430079 \\ ${ }^{2}$ School of Foreign Languages, Fuyang Teachers College, Fuyang, Anhui, 236041 \\ yy7589@sina.com
}

Keywords: Semantic attrition; adverbs of high degree; semantic compensation; NLP.

\begin{abstract}
The thesis studied the semantic attrition of traditional degree adverbs based on the natural language processing. The usage of new-generation adverbs of high degree such as "bao, chao, ju, kuang, qi” is explored in detail. Finally, the semantic compensation mechanism was also briefly studied.
\end{abstract}

\section{Introduction}

Zhu Dexi(1982)wrote in his Lecture Notes on Grammar in this way: the grammatical function of the degree adverbs is to modify the adjectives and some verbs and certain kind of verb phrase. As for the classification standards and methods of the degree adverb, Wang $\mathrm{Li}(1985)$ is more influencing and accepted by more people, who divided it into two kinds, one is the absolute degree adverb and the other is the relative degree adverb; Based on that, Zhou Xiaobing(1995)further divided the absolute degree adverb into three subclasses as follows: extreme degree, high degree and low degree. Although degree adverbs are not so much in Mandarin Chinese, they are used highly frequently in the daily communication and writing and maybe because of that, their semantic attrition is also high. When the original meaning of degree cannot be properly expressed, the new expressions are needed to be created.

\section{Semantic attrition}

Semantic attrition is one kind of language attrition. The semantic attrition studied in this thesis is different from the hot topics of language attrition in the field of foreign language although they are interrelated with each other in some aspects.

Language attrition, as an independent studying field, was begun with the first seminar of "the Loss of Language Skill” in the Pennsylvania University in 1980. Thirty years after that, the study of language attrition has been developed and achieved a lot, especially in the attrition study of the first language acquisition in the environment of the foreign language and the attrition study of the foreign language acquisition in the environment of the first language, contributing a great deal to the FLA and SLA and having a deep influence over them. Interestingly, there is not a universally accepted definition of the language attrition. Li Xiukui and Ren Huiqin(2011)defined it as the loss or the degeneration of the skill and knowledge about the second language or the foreign language because of not using them after some time of learning. Yang Lianrui(2011)considered the language attrition refers to the letdown or loss of the language application ability for the bilingual or multilingual individuals or groups because of the reduction or stop of using.

From the above definitions, we can see that they focus mainly on the phenomena of forgetting or reducing or stopping of the learners' language ability in different levels (phonetics, vocabulary, semantics and pragmatics, etc.) during the process of language acquisition(including the FLA and SLA). But the semantic attrition discussed in this thesis refers to the wastage, reduction and loss of the original meaning with regard to its quality and quantity during the long-term employment with high frequency, just as the using of the stuff or article. If you make use of it for a long time, its function will be reduced even lost, so the necessary maintenance, repair, change of the spare parts 
even the change of the whole stuff, updated by the new stuff are needed. Language is like the stuff, obeying the natural law that using makes the thing wear down and idling makes it degenerate, so if certain expression was used for a long time, it also need to be maintained or repaired even changed by the new generation. As far as the degree adverb is concerned, its original aim is to emphasize the high degree, but if you use the same "hen, ting, feichang"every time, the aesthetic tiredness or weariness is certain to take place: the first time when you hear the sentence "he is very angry", you are so deeply impressed; the second time you have a relative deep impression, but how about the third time, the fourth time, let alone a thousand times and a million times, you can not feel the high degree at all. In other words, the meaning of "feichang" was worn down during the frequent use and its function is greatly reduced, and at this time, the second generation "product" was desperately needed to replace the old one.

\section{“bao, chao, ju, kuang, qi”-New Adverbs of High Degree}

The employment of the traditional high degree adverb with high frequency makes their modifying ability decline. When these traditional expressions cannot fully reflect the degree of their behavior or state, so the new-generation adverbs of high degree 1 such as "bao, chao, ju, kuang, qi" are widely used in the oral communication of the young man, at first mainly online, later, they come to penetrate into the written style.

\section{"bao"}

From our online searching statistics, the employment frequency of "bao" is not very high. When modified adjective, "baoqiang (extremely surprising) is the most frequent, while modified the verb, "baoda/bao’ou/baoji (barbarously beat somebody)", "baozhang (sharply increase)", "baokou (violently dumping)" are used more frequent than others:

(1)ren kending baoduo.(There must be extremely crowded.) $)^{2}, 3$

"chao"

As a new-generation adverb of high degree, "chao" is used most widely among the five. Almost all the adjective can collocate with it and this word appeared so often in the newspaper, magazine, broadcast, TV and literature to such a degree that it is overused or we can say it is also in attrition.

(2)Wuliu chaokuai, (The transportation is very fast.)

Originally, "chao" mainly modified monosyllable adjective and later it pervaded to bisyllable words.Modification the trisyllable words with "chao" is rare:

(3)Shibushi meige nansheng youdian ziben jiuyiwei ziji chaoliaobuqi le?

(Is it right that the boys who possess some talent think themselves super great?)

“ju”

The statistics of our online searching show that the employment frequency of "ju” is lower than "bao", another difference is that "ju” mainly modifies the adjectives of two syllables.

(4)ju BT(biantai) wenti, nineng caichu jige! (How many questions can you guess among these highly abnormal ones?)

\section{"kuang”}

\footnotetext{
${ }^{1}$ Zhou Juan(2006)analyzed this kind of adverb through four aspects: their collaboration function, formation basis, pragmatic value and development tendency and called them "bao"-type new popular adverbs of high degree. But Hu Lizhen(2008)hold that this view should be reconsidered and the opinion that this kind of adverb appeared in twenty-first century is wrong because their adverb usage did exist in the ancient Chinese; With a lot of examples, she proved that their popularity today is only "the activation and inheritance of their ancient meanings". We agree with Hu Lizhen(2008)and named this kind of expression "new-generation adverbs of high degree" to make a difference with them and hold that they are not new words, but the prominence of certain item of meaning of the common words.

${ }^{2}$ The examples in this thesis, except those with special explanation, are mainly from different websites, BBS, bars, public talking rooms and the Weibo in sina. Sometimes there are some mistakes in the examples from the internet; we made some explanation or correction in the brackets following the examples for the readers' easy understanding.

${ }^{3}$ Here we did not use the traditional three-line model of "example, word-to-word translation, liberal translation" because we only focus on the semantics, not the grammatical function difference.
} 
"kuang" is common to used as a noun in Mandarin Chinese, such as "gongzuokuang(a workaholic), gouwukuang(a shopaholic), luedaikuang(sadism)"etc. Actually, it is not rare for "kuang”to use as an adverb of high degree,for example:kuangxi, kuangre, kuangnu, kuanghuan, kuangben, kuangpai, kuangma, kuangkan, kuangyin baoshi etc. As a new-generation adverb of high degree, "kuang" mainly modifies the verb. Sometimes "kuang"is used in the title and advertisement. While It is really rare for "kuang” to modify the adjective.

(5) ta chanhou kuangshou 9 gongjin

(She crazily lost 9 kilograms after giving birth.)

(6) aoteman kuangbian xiaoguaishou(youxi ming)

(Automan violently beats the little monster. (a game name))

“qi”

Among the five adverbs discussed above, “qi” is the one which is employed least, but its usage as a degree adverb is with a long history in Chinese. The following are the household words including it: "qiqiang, qixian, qiqiao, qichouwubi (which is a productive construction and the position of "chou" can be replaced by many words.) etc. More examples from the internet:

(7) diannao kaiji sudu qiman/kuai.

(The opening speed of the computer is especially slow/fast.)

\section{Semantic Compensation for Attrition}

Language is in the dynamic and developing state. Its direct developing impetus is the development of the politics, economy and the culture, the progress of the society, the appearance of the new things and the mental need and way of thinking for the new and different. "hen, ting, feichang, shifen" were used so frequently and people are familiar with it just as his palm, which directly led to their semantic attrition, so people began to find new ways to make up for it. Such as the duplication of the adverb: wo feichang feichang gaoxing. (I am very very happy.) and wo feichang feichang feichang gaoxing.(I am very very very happy.)

Or the overuse the high degree adverb, that is, to use it to modify the adjective which cannot modify in the past:hen binglen,feichang binglen, shifen binglen.(very cold) and feichang xuebai (very white as the snow).

Or the addition or improvement the number in the original expressions:

(8) wo shifen de manyi.(I am ten tenth satisfactory.)

(9) wo shi'erfen de manyi.(I am twelve twelfth satisfactory.)

(10) wo baifenbai de manyi.(I am 100 percent satisfactory.)

(11) wo wanfen de manyi.(I am satisfactory a thousand and one.)

(12) wo shi'erwanfen de manyi.(I am satisfactory one hundred twenty thousand and one.)

(13) wo ershiwanfen de manyi.(I am satisfactory two hundred thousand and one.)

When attrition happened to all of these expressions, the new-generation adverbs of high degree such as "bao, chao, ju, kuang, qi” were desperately required to appear to shoulder the task of correctly and vividly expressing people's ideas

We have observed that these new-generation adverbs of high degree themselves are also in the state of attrition because the language is like the cake, which has a time limit of keeping fresh. The high frequency loaded in these words came to waste and wear down after long-term employment, so the new compensation mechanism is on the way:

New-generation adverbs of high degree coexist, that is, to use two or more of them together at the same sentence or mixed with each other.

(14)suoyouren dou juchangkuang jukuazhang de daxiao.( zhuanyinzi Zhou juan)

(All the people are laughing furiously and turgidly. (from Zhou Juan(2006))

(15) qiu chaoji juhaoting yingwen gequ/chaoji juganren dianying!

(Ask for some super fair-sounding English songs/ super especially moving movies!)

It is not hard to imagine that in the near future, the new-generation adverbs of high degree will also suffer from the attrition because of the high frequency till the new better expression was created to 
replace them and enter the historical stage of language. It is the metabolism, development, renewal and change time and time again that push the language to the subtle and the perfect.

\section{Conclusions}

Language is forever in the dynamic and developing state. The traditional adverbs of high degree "hen, ting, guai, geng, zui, tai, tui, hao, zhen, feichang, shifeng, tebie, youqi, etc" are seriously worn down because of the employment with high frequency and is replaced slowly by the new-generation adverbs of high degree such as "bao, chao, ju, kuang, qi" in oral communication, written material, in the virtual world or in the real life, regardless of the fact that you are willing to accept them or not, while the changes do happen everyday.

Attrition contradicts with the compensation; meanwhile, they help each other and complement each other to develop together. It is possible that the new-generation adverbs of high degree such as "bao, chao, ju, kuang, qi" will only appear for a very short time and will be replaced by other better expressions and finally fade out of our horizon, and will be fallen into oblivion and gone with the wind. But now, they are prosperous and developing healthily and proudly, just like the rain fallen in the night with the wind, irrigating everything silently and thoroughly. Its frequent appearance in the internet and all kinds of media makes us to pay attention to it. Facing these new language phenomena, we'd better tolerate and respect them and take an open-minded and enlightened attitudes towards them, and then, we are willing to pay some attention to them and try to understand them even at last we will make use of them consciously or unconsciously. As for our linguists, we should try our best to observe and explain them and lead them to a more healthy and standard development, contributing a lot to the natural language processing and the social development.

\section{References}

[1] Hu, Lizhen. On Three Degree Adverb “ju” 、 “kuang”、 “qi”[J]. xiuci xuexi,2008(3): 79-80.

[2] Lv, Shuxiang. 800 words of Contemporary Chinese[M].Beijing:the Commercial Press,1999.

[3] Ni, Chuanbin. Essential Attributes of Foreign Language Attrition [J]. Waiguoyu,2007(1): 42-50.

[4] Yang, Lianrui. Social Psychological Factors of the Foreign Language Attrition Studies [J]. waiyu jiaoxue, 2011(2): 40-43.

[5] Wang, Li. Modern Chinese Grammar [M]. Beijing:the Commercial Press,1985. [6]Wu, Lihong. The Degree Attrition of the State Adjective and its Expression Change[J]. xiuci xuexi,2005(6): 19-22. [6] Zhang, Ge. The Reviews, Problems and Prospects of the Degree Adverb Studies in Mandarin Chinese [J]. sixiang zhanxian,2008(1): 110-114.

[7] Zhou, Juan. The Multi-dimensional Studies of “bao”-type New Popular Adverbs of High Degree [J]. xiuci xuexi,2006(6): 45-48.

[8] Zhou, Xiaobing. On the Degree Adverb in Mandarin Chinese [J]. zhongguo yuwen,1995(2): 100-104.

[9] Zhu, Dexi. Lecture Notes on Grammar [M]. Beijing:the Commercial Press,1982. 